\title{
Are we dealing with hearing-impaired children correctly?
}

\author{
Dental care for children and young people who have a hearing impairment by J. Champion and R. Holt \\ Br Dent J 2000; 189: 155-159
}

\begin{abstract}
Aim
The aim was to determine whether there are indications that hearing-impaired children experience difficulties in accessing dental care and/or in receiving dental treatment.

\section{Method}

The study was carried out by means of a questionnaire. Parents of 84 children contacted through the National Deaf Children's Society returned completed questionnaires.

\section{Results}

Eighty-two children (98\%) had visited a dentist. Nearly twothirds $(63 \%)$ were reported to have at least one problem in communication while receiving dental care, this increased significantly as the severity of the hearing impairment increased. Fifty-nine children $(70 \%)$ reported having at least one problem in communication at the doctors. Fifty-two (62\%) reported that the dentist had worn a mask while communicating with the child and $48(57 \%)$ that there had been background noise in the surgery during appointments.
\end{abstract}

\section{Conclusions}

Removing masks while talking, reducing background noise and learning to use simple signs may improve communication with hearing-impaired children.

\section{In Brief}

- Remove masks while communicating with the child and reduce background noise during the appointment by switching off music or closing the window

- Devise a deaf-friendly call system such as the dentist going into the waiting room to take the child to the surgery

- Learn a few basic signs, be prepared to write down essential information and use picture books to explain procedures

- When communicating with a child, be sure to face the child, the right way up and ensure the light is not in the child's eyes or behind your head

- Install a minicom or, alternatively, familiarise the staff with Typetalk services

\section{Comment}

'Wow can I communicate with this dentist when I am deaf and he's wearing a mask?' So say $62 \%$ of children in this report of a questionnaire survey of the parents of 84 children who were hearingimpaired.

Somewhere between 23,000 and 25,000 children in the UK are permanently deaf or hard of hearing. The majority are born deaf or acquire a hearing impairment early in life. The aim of this survey was to determine whether hearing-impaired children experience difficulties in accessing dental care and/or in receiving treatment. The paper very helpfully details the different communication methods available for use by people with a hearing impairment.

The study was carried out with the help of the National Deaf Children's Society who arranged for the distribution of the questionnaire through their regional network.
The results may therefore not be fully representative of all deaf or hearing-impaired young people in the UK.

The majority of children, 82 out of the 84 , were reported to have attended a dentist, with 70 having done so before 5 years of age. Children with a profound hearing loss were less likely to have visited a dentist before 5 years or to have received treatment of any sort than their less severely affected peers. Only 10 families reported difficulties in obtaining a dental appointment for their child - and in only two cases was this overtly because of communication difficulties. However, for those who saw a dentist, 53 children had difficulties with problems in communication being the commonest. Amazingly, 27 parents reported that the dentist always wore a mask and a further 25 said that the dentist sometimes wore a mask.
The paper, very positively, reports examples of good practice that were either experienced or were desirable at the dentist; most of these relate to aids in communication like text telephones, explanatory booklets and videos. One very salutary point was the unease parents felt at having to be present as an interpreter for their child who was reaching an age when they felt that their presence was an intrusion of privacy.

Many of these recommendations have only limited resource implications. As we are members of the caring professions I think we should seriously consider doing more to enhance our communication skills for this not insignificant group in the population.

\section{June H Nunn}

Department of Child Dental Health, Dental School, University of Newcastle upon Tyne 